\title{
Semi-discrete solitons in arrayed waveguide structures with Kerr nonlinearity
}

\author{
N.-C. Panoiu, ${ }^{1}$ B. A. Malomed, ${ }^{2}$ and R. M. Osgood, Jr. ${ }^{3}$ \\ ${ }^{1}$ Department of Electronic and Electrical Engineering, \\ University College London, Torrington Place, London WC1E 7JE, UK \\ ${ }^{2}$ Department of Physical Electronics, Faculty of Engineering, Tel-Aviv University, Tel-Aviv 69978, Israel \\ ${ }^{3}$ Department of Applied Physics and Applied Mathematics, \\ Columbia University, New York, New York 10027, USA
}

(Dated: July 25, 2021)

\begin{abstract}
We construct families of optical semi-discrete composite solitons (SDCSs), with one or two independent propagation constants, supported by a planar slab waveguide, XPM-coupled to a periodic array of stripes. Both structures feature the cubic nonlinearity and support intrinsic modes with mutually orthogonal polarizations. We report three species of SDCSs, odd, even, and twisted ones, the first type being stable. Transverse motion of phase-tilted solitons, with potential applications to beam steering, is considered too.

PACS numbers: 42.65.Tg, 42.65.Wi, 42.65.Jx, 42.65.Sf, 42.79.Gn, 42.82.Et.
\end{abstract}

Power exchange among evanescently coupled cores in arrays of single-mode nonlinear waveguides has attracted a great deal of interest, because such interactions reveal new phenomena in the wave dynamics in discrete systems, and due to their potential applications to alloptical switching (see Ref. [1] for a review of the light transmission in linear and nonlinear discrete systems). It was predicted that such lattices support discrete solitons $[2,3,4]$, i.e., localized modes for which the discrete diffraction in the waveguide array balances the onsite cubic $\left(\chi^{(3)}\right)$ nonlinearity, and more complex modes, such as discrete vortex solitons [5] (in two-dimensional lattices), discrete surface solitons [6] and light bullets [7]. In addition, the existence of continuous solitons in semi-discrete nonlinear media based on Bragg optical fibers have also been recently predicted [8]. The discrete solitons, as well as their counterparts predicted in $\chi^{(2)}$ media [9], have been observed in experiments $[10,11,12]$.

In recent work [13], it was demonstrated that, if a periodic array of optical waveguides is nonlinearly coupled to a slab waveguide, both structures being made of a material with quadratic $\left(\chi^{(2)}\right)$ nonlinearity, the combined optical structure supports a new species of optical solitons, namely semi-discrete composite solitons (SDCS). These solitons are different from their fully discrete and continuous counterparts in several aspects; in particular, it has been shown that both even (symmetric) and twisted (antisymmetric) SDCS may be stable.

In this paper, we extend those ideas to the obviously relevant case of composite structures made of optical media with cubic nonlinearity. We demonstrate, for the first time to our knowledge, that such media support oneand two-parameter families of stable SDCSs, which consist of two mutually trapped components, viz., a discrete one, which is chiefly carried by the waveguide array, and a continuous component, bound to the slab waveguide. We also demonstrate that such SDCS can be used in optically controlled beam steering devices, and therefore they could have important practical applications.

We consider the structure built as a periodic array of stripe optical waveguides, whose adjacent cores are separated by a distance $d_{0}$, which is coupled to a singlemode slab waveguide. The waveguide array may be either buried into the slab waveguide, as shown in Fig. 1, or mounted on top of it. Both the stripes and the slab are to be made of a Kerr material, possible choices being silicon-on-insulator (SOI) [14], polymers [15], or semiconductor heterostructures [16]. We also assume that the structure is designed so as to make the intrinsic modes in the stripe and slab waveguides polarized orthogonally to each other, thus ruling out the linear coupling between them. Specifically, if we consider buried SOI waveguides, by simply changing the transverse dimensions of the silicon waveguide one can design stripe waveguides which support either TE or TM polarized modes [17], and therefore an experimental set up involving orthogonally polarized modes can be readily implemented. In the experiment, the incident wide beam may be unpolarized, the stripe and slab waveguides picking mutually orthogonal polarization components from it. As a result, the propagating mode in each stripe is linearly coupled to its counterparts in the adjacent ones, and nonlinearly coupled, through the cross-phase modulation (XPM), to the slab mode. In addition, the case of linear coupling between a transversely confined waveguide mode and a slab waveguide mode has previously been considered in Ref. [18] and therefore it would not be discussed here.

Coupled-mode equations for optical fields in the composite medium can be derived in a consistent form

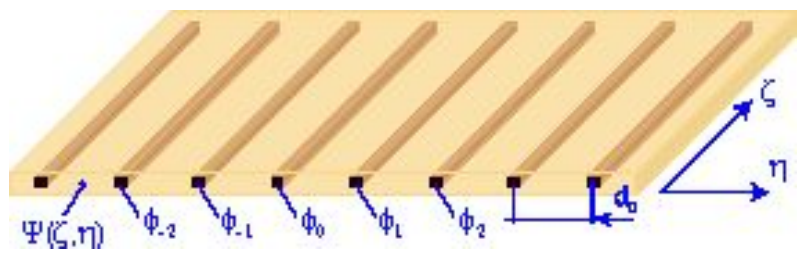

FIG. 1: (Color online) A schematic of the composite optical structure. 
$[19,20]$, resulting in a system of ordinary differential equations for the discrete component, coupled to a partial differential equation governing the propagation of the slab mode,

$$
\begin{gathered}
i \frac{d \phi_{n}}{d \zeta}+\bar{\beta}_{d} \phi_{n}+\phi_{n-1}+\phi_{n+1}+\phi_{n}\left|\phi_{n}\right|^{2} \\
+\kappa_{d} \phi_{n}|\Psi(\zeta, \eta=n)|^{2}=0, \\
i \frac{\partial \Psi}{\partial \zeta}+\bar{\beta}_{c} \Psi+\frac{1}{2} \frac{\partial^{2} \Psi}{\partial \eta^{2}}+\Psi|\Psi|^{2} \\
+\kappa_{c} \Psi \sum_{n}\left|\phi_{n}\right|^{2} \delta(\eta-n)=0 .
\end{gathered}
$$

Here, $\bar{\beta}_{c, d}=\beta_{c, d} / c_{d}$ are normalized wavenumbers, with $\beta_{c}\left(\beta_{d}\right)$ the propagation constant in the slab (stripe) waveguide in physical units, and $c_{d}$ the constant of the linear coupling between the stripe modes, while $\zeta=z / z_{0}$ and $\eta=x / x_{0}$, where $z$ and $x$ are the longitudinal and transverse coordinates, and $z_{0} \equiv 1 / c_{d}$, $x_{0} \equiv\left(\beta_{c} c_{d}\right)^{-1 / 2}$. The normalized fields $\phi_{n}$ and $\Psi$ are rescaled modal fields $u_{n}$ and $U$ (which are measured in units of $\sqrt{\mathrm{W}}$ and $\sqrt{\mathrm{W} / \mathrm{m}}$, respectively): $\phi_{n}=\sqrt{\gamma_{d} / c_{d}} u_{n}$ and $\Psi=\sqrt{\gamma_{c} / c_{d}} U$. Finally, $\kappa_{d}$ and $\kappa_{c}$ are relative strengths of the XPM and SPM (self-phase modulation) interactions, $\kappa_{d}=\sqrt{\gamma_{d c} / \gamma_{c}}, \kappa_{c}=\sqrt{\gamma_{c d} / \gamma_{d}}$. Here, coefficients $\gamma$ are defined as integrals over cross sections of the respective waveguides $\left(A_{d}\right.$ and $A_{c}$, the latter defined as the transverse area of the slab waveguide between adjacent stripes), involving the convolution of the third-order susceptibility tensor, $\hat{\chi}^{(3)}$, with the stripe and slab modes, $\mathrm{e}_{d}(x, y)$ and $\mathrm{e}_{c}(y)$, which are normalized to the corresponding mode powers, $\mathcal{P}_{d}$ and $\mathcal{P}_{c}$ (measured in $\mathrm{W}$ and $\mathrm{W} / \mathrm{m}): \gamma_{d}=\left(3 \epsilon_{0} \omega / 4 \mathcal{P}_{d}^{2}\right) \int_{A_{d}} d A \mathrm{e}_{d}^{*}$. $\hat{\chi}^{(3)}: \mathrm{e}_{d} \mathrm{e}_{d}^{*} \mathrm{e}_{d}, \quad \gamma_{c}=\left(3 \epsilon_{0} \omega / 4 \mathcal{P}_{c}^{2} d_{0}\right) \int_{A_{c}} d A \mathrm{e}_{c}^{*} \cdot \hat{\chi}^{(3)} \mathrm{e}_{c} \mathrm{e}_{c}^{*} \mathrm{e}_{c}$, $\gamma_{d c}=\left(3 \epsilon_{0} \omega / 2 \mathcal{P}_{d} \mathcal{P}_{c}\right) \int_{A_{d}} d A \mathrm{e}_{d}^{*} \cdot \hat{\chi}^{(3)}:_{\mathrm{e}_{d}} \mathrm{e}_{c}^{*} \mathrm{e}_{c}$, and $\gamma_{c d}=$ $\left(3 \epsilon_{0} \omega / 2 \mathcal{P}_{d} \mathcal{P}_{c} d_{0}\right) \int_{A_{c}} d A \mathrm{e}_{c}^{*} \cdot \hat{\chi}^{(3)}: \mathrm{e}_{c} \mathrm{e}_{d}^{*} \mathrm{e}_{d}$. The same system of coupled-mode equations may also be interpreted as the one governing the transmission of waves $\phi_{n}$ and $\Psi$ with different carrier frequencies, which also rules out the linear coupling between them.

To focus on the most fundamental case, we assume that the stripe and slab modes are phase-matched, $\bar{\beta}_{d}=$ $\bar{\beta}_{c} \equiv \bar{\beta}$, hence the corresponding linear terms in Eqs. (1) can by removed (this condition can be easily satisfied by adjusting geometrical parameters of the stripe and slab), and the nonlinear coupling between the stripe and slab waveguides is symmetric, with $\kappa_{d}=\kappa_{c} \equiv \kappa$. Then, Eqs. (1) conserve the Hamiltonian,

$$
\begin{aligned}
H & =-\sum_{n}\left(\phi_{n}^{*} \phi_{n+1}+\phi_{n} \phi_{n+1}^{*}\right)-\frac{1}{2} \sum_{n}\left|\phi_{n}\right|^{4} \\
& +\frac{1}{2} \int_{-\infty}^{+\infty}\left(|\partial \Psi / \partial \eta|^{2}-|\Psi|^{4}\right) d \eta-\kappa \sum_{n}\left|\phi_{n} \Psi(n)\right|^{2}
\end{aligned}
$$
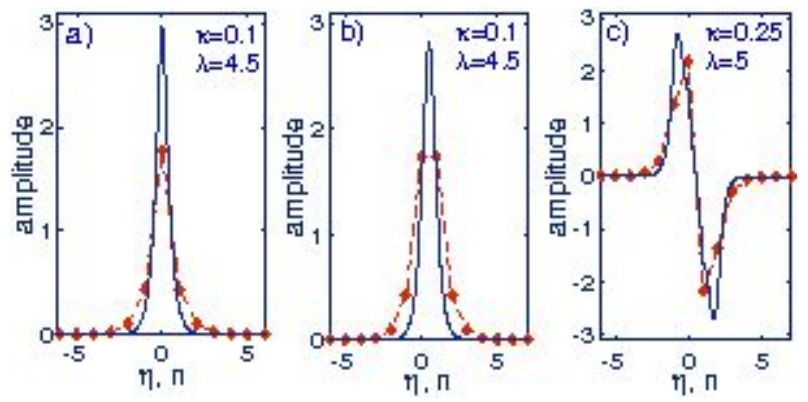

FIG. 2: (Color online) Field profiles of the three species of the semi-discrete composite solitons: odd, alias onsite-centered (a); even, alias intersite-centered (b); and twisted (c).
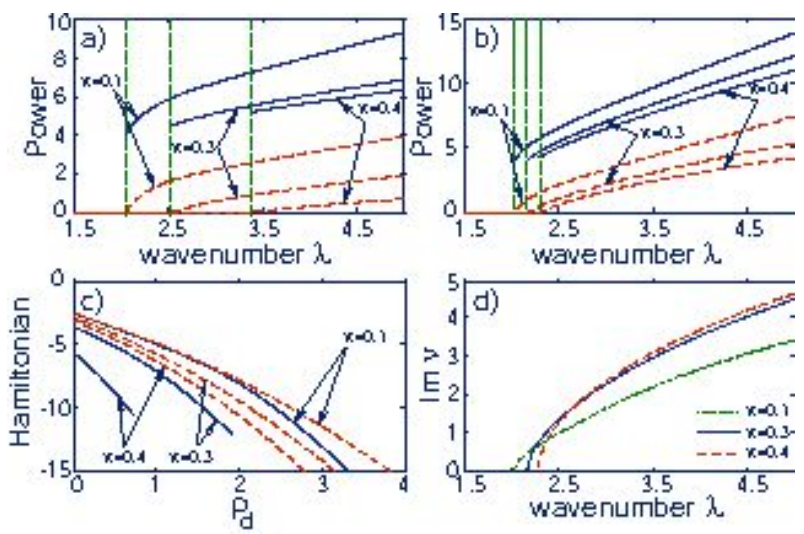

FIG. 3: (Color online) Top panels: total power $P$ and power $P_{d}$ of the discrete component (solid and dashed lines, respectively) versus $\lambda$, for (a) odd and (b) even solitons. (c) Hamiltonian $H$ of the odd and even solitons (solid and dashed lines, respectively) versus $P_{d}$. (d) The dominant instability growth rate for even solitons versus $\lambda$.

(the asterisk stands for the complex conjugation), and total powers of the discrete and continuous components, $P_{d}=\sum_{n}\left|\phi_{n}\right|^{2}$ and $P_{c}=\int_{-\infty}^{+\infty}|\Psi|^{2} d \eta$, respectively.

General soliton solutions to Eqs. (1) can be looked for as $\phi_{n}(\zeta)=\bar{\phi}_{n} e^{i \lambda_{d} \zeta}$ and $\Psi(\eta, \zeta)=\bar{\Psi}(\eta) e^{i \lambda_{c} \zeta}$, with $\lambda_{d} \neq \lambda_{c}$, i.e., they form a two-parameter family of solitons $[21,22,23]$. To find these solutions, we numerically solved the respective stationary version of Eqs. (1), thus constructing both a particular one-parameter set of the solutions, with $\lambda_{d}=\lambda_{c} \equiv \lambda$, and the general twoparameter family of SDCSs $\left(\lambda_{d} \neq \lambda_{c}\right)$.

Figures 2(a)-(c) display generic examples of the solitons belonging to the one-parameter families of the odd, even, and twisted types, obtained through this procedure (the former two may also be classified as onsite-centered and intersite-centered, respectively, as concerns their discrete component). The entire families are presented in Figs. 3(a)-(c) by plots showing Hamiltonian $H$, total power $P \equiv P_{c}+P_{d}$, and the power in the discrete component, $P_{d}$, as functions of common wavenumber $\lambda$. Both odd and even solitons exist only if $\lambda$ exceeds a threshold value, $\lambda_{\text {thr }}$, which increases with the coupling strength, 

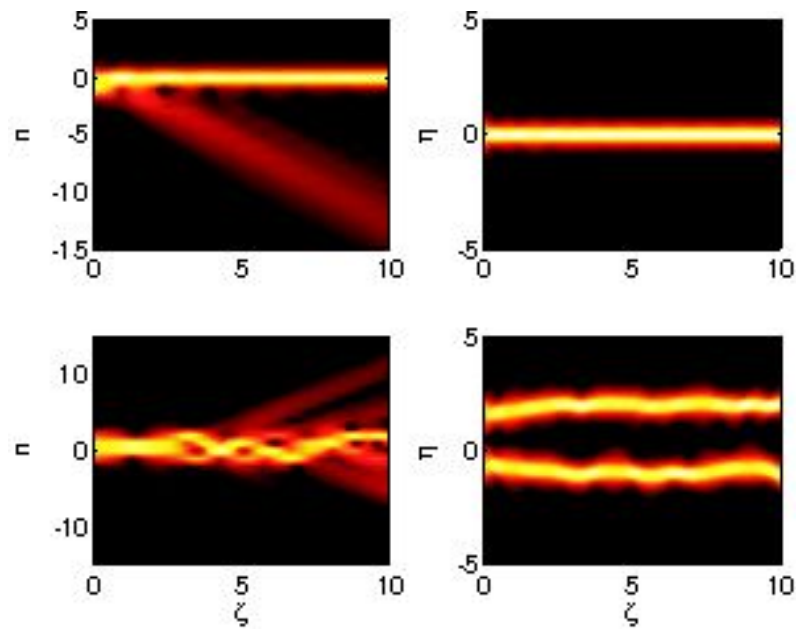

FIG. 4: (Color online) Unstable propagation of even (top panels) and twisted (bottom panels) semi-discrete composite solitons. Left and right panels correspond to the discrete and continuous components, respectively. The soliton parameters are the same as in Fig. 2.

$\kappa$. This result can be explained by the dispersion relation for CW solutions to Eq. (1b), with amplitude $\Psi_{\mathrm{cw}}$ and transverse wavenumber $k_{0}, \lambda_{\mathrm{cw}}=2 \cos k_{0}+\kappa\left|\Psi_{\mathrm{cw}}\right|^{2}$ : since nonlinear modes exist for $\lambda>\lambda_{\mathrm{cw}}$ only, $\lambda_{\mathrm{thr}}$ indeed increases with $\kappa$. Moreover, diagram $\left(H, P_{d}\right)$ in Fig. 3(c) suggests that the odd (onsite-centered) composite solitons may be stable, as they correspond to a smaller value of $H$, and become more localized as $P_{d}$ increases. Indeed, note that the Peierls-Nabarro barrier (PNB), which is defined as the difference in $H$ of odd and even solitons that have the same power $P_{d}$ [24], increases with $P_{d}$.

The expectations concerning the stability of the SDCSs are supported by the linear-stability analysis. To perform the analysis, we linearized Eqs. (1) around the stationary soliton solutions, $\bar{\phi}_{n}^{(\mathrm{sol})}$ and $\bar{\Psi}^{(\mathrm{sol})}$, setting $\phi_{n}=\bar{\phi}_{n}^{(\text {sol })}+\left(\delta \phi_{n} e^{i \nu \zeta}+\delta \bar{\phi}_{n}^{*} e^{-i \nu^{*} \zeta}\right) e^{i \lambda \zeta}$ and $\Psi=$ $\bar{\Psi}^{(\mathrm{sol})}+\left(\delta \Psi e^{i \nu \zeta}+\delta \bar{\Psi}^{*} e^{-i \nu^{*} \zeta}\right) e^{i \lambda \zeta}$, and calculating eigenvalues $\nu$ from the ensuing linearized equations for the small perturbations. The results, summarized in Fig. $3(\mathrm{~d})$, demonstrate that all even solitons are unstable, which is natural to states whose discrete component is centered at the intersite position [1]. Twisted SDCSs are unstable too, in their entire domain of existence. On the contrary, odd SDCSs are completely stable, as $\operatorname{Im}(\nu)=0$ for all $\lambda$ at which they exist.

A generic scenario for the unstable propagation of even and twisted SDCSs is illustrated in Fig. 4, the input solitons being those presented in Fig. 2. Thus, in the case of even SDCSs, the discrete component evolves into an odd discrete soliton, which then becomes locked in with the continuous component. As a result, an odd SDCS is formed. Moreover, during this process part of the energy contained in the initial soliton is released as radiation. On the other hand, twisted SDCSs break in a pair of mutually trapped odd solitons, which continuously emit
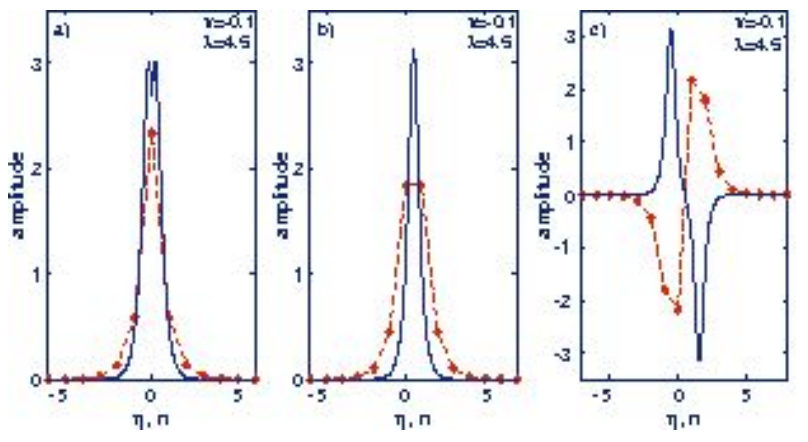

FIG. 5: (Color online) Field profiles of the three species of the semi-discrete composite solitons: odd, (a); even, (b); and twisted (c), corresponding to the case $\kappa<0$.
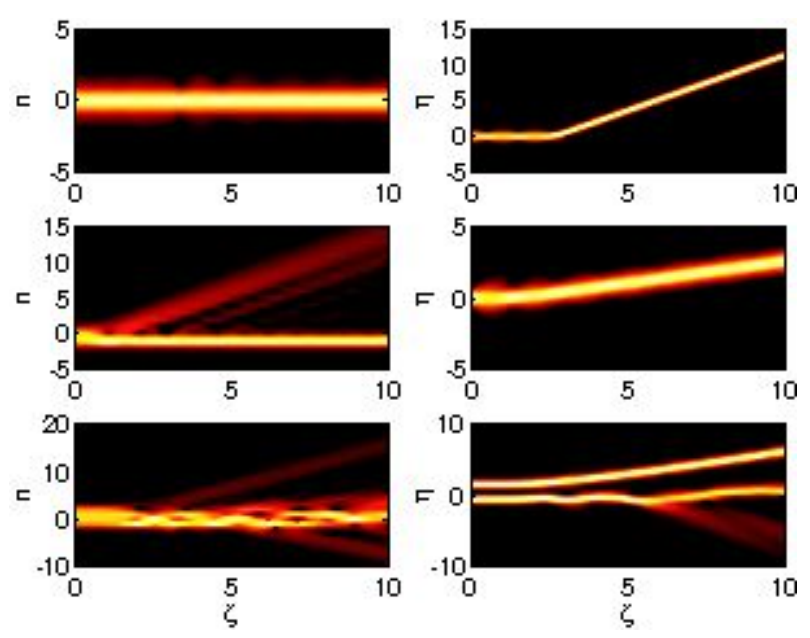

FIG. 6: (Color online) Unstable propagation of odd (top panels), even (middle panels), and twisted (bottom panels) semidiscrete composite solitons. Left and right panels correspond to the discrete and continuous components, respectively. The soliton parameters are the same as in Fig. 5.

radiation as they copropagate.

For the sake of completeness of the study of the model (1), we also investigated the existence and stability of soliton solutions in a more formal case, namely that of $\kappa<0$. Generic odd, even, and twisted soliton solutions are shown in Fig. 5. Our analysis shows that the main difference between this case and the case in which $\kappa>0$ is that for $\kappa<0$ all three species of solitons are unstable. Thus, as our numerical simulations illustrate, if $\kappa<0$ odd SDCSs are unstable, too, upon a small transverse perturbation in the field profile. Specifically, due to the self-defocusing characteristics of the XPM interaction in this case, the discrete and continuous components repel each other and form discrete and continuous solitons, which separate from each other upon propagation (see Fig. 6, top panels). This soliton break up is also observed in the case of even and twisted SDCSs.

General two-parameter SDCS families of the odd and even types, with $\lambda_{d} \neq \lambda_{c}$, have also been found numerically as stationary localized solutions of Eqs. (1). The 

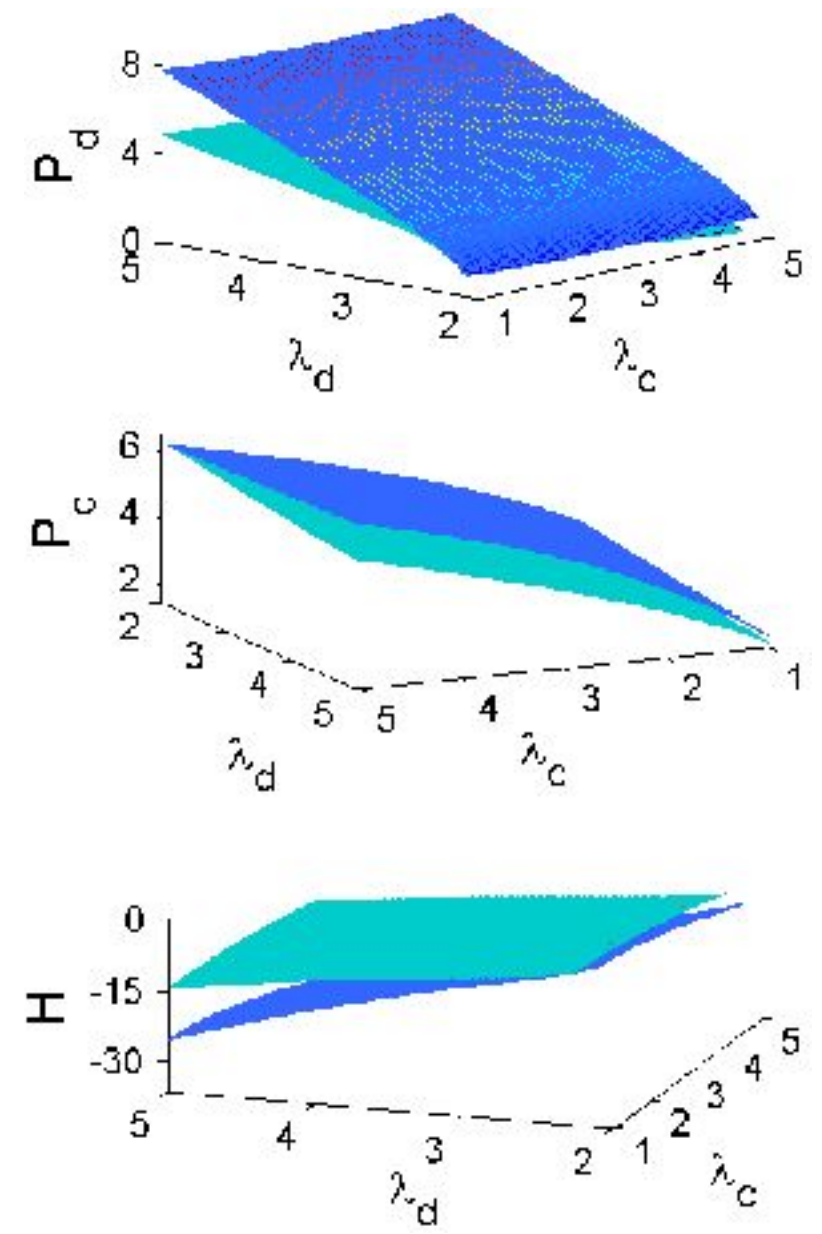

FIG. 7: (Color online) The power in the discrete and continuous components (middle and top panels, respectively) of the general soliton family versus independent wavenumbers $\lambda_{d}$ and $\lambda_{c}$. The dependence of Hamiltonian $H$ on $\lambda_{d}$ and $\lambda_{c}$ is shown in the bottom panel. In all panels, the nonlinearcoupling constant is $\kappa=0.1$, the blue and green (upper and lower) surfaces corresponding to the odd and even SDCS, respectively.

results of these calculations, performed for both odd and even SDCSs, are presented in Fig. 7, through the respective dependences of the dynamical invariants, $P_{d}, P_{c}$ and $H$, on $\lambda_{d}$ and $\lambda_{c}$. The first among these dependences shows that, similar to the case of $\lambda_{d}=\lambda_{c}=\lambda$, SDCSs exist only if $\lambda_{d}$ exceeds a certain threshold value.

To analyze the stability of the general SDCS family, we first used an extension of the Vakhitov-Kolokolov (VK) criterion [25] for two-parameter families of solitons [26, $27,28]$, according to which the stability changes across curves $J=0$ in the parameter space, where the Jacobian is given by the following expression:

$$
J=\frac{\partial\left(P_{d}, P_{c}\right)}{\partial\left(\lambda_{d}, \lambda_{c}\right)}=\frac{\partial P_{d}}{\partial \lambda_{d}} \frac{\partial P_{c}}{\partial \lambda_{c}}-\frac{\partial P_{d}}{\partial \lambda_{c}} \frac{\partial P_{c}}{\partial \lambda_{d}} .
$$

Using the computed powers $P_{d}$ and $P_{c}$, we have concluded that $J \neq 0$ (in fact, $J>0$ ) for all values of $\lambda_{d}$ and
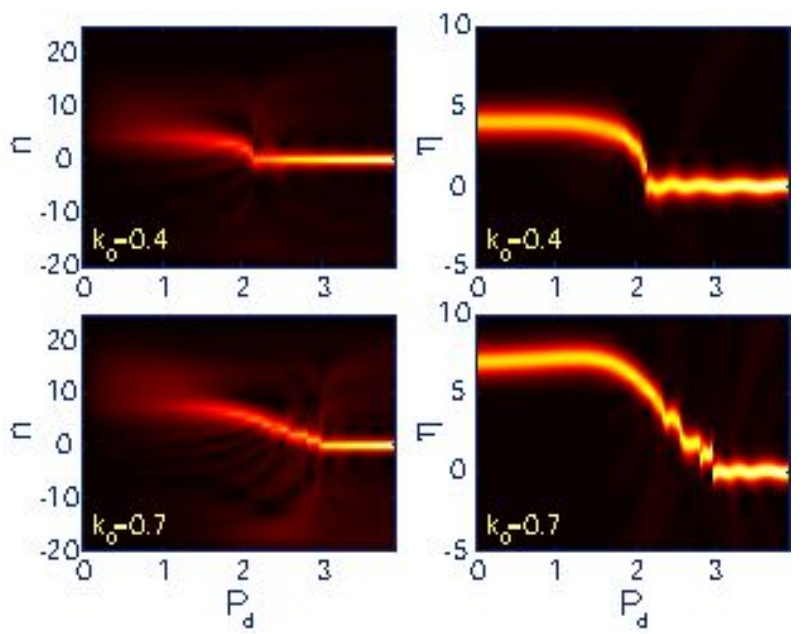

FIG. 8: (Color online) Contour plots of the discrete and continuous field components (left and right panels, respectively) of odd (stable) solitons subjected to the initial phase tilt, $k_{0}$. In this and following figures, the profiles were recorded after propagation distance $\zeta_{0}=10$, for coupling constant $\kappa=0.1$. At $P_{d}<1$, the discrete component is spread over several stripe waveguides, a transition to pinning being clearly seen at $P_{d} \approx 2$ and 3 , for $k_{0}=0.4$ and 0.7 , respectively. Note that the continuous component is stronger localized than the discrete one.

$\lambda_{c}$ at which SDCSs exist; therefore, both odd and even SDCSs do not change the VK stability in their existence domains.

Because the VK criterion is only a necessary stability condition [note that it does not predict the instability of the one-parameter family of even SDCSs, see Fig. 3(b)], we have also performed the full linear-stability analysis for the two-parameter families, and concluded that (as might be expected), the odd solitons are stable, whereas their even counterparts are not. These conclusions accord to the topology of surface $H\left(P_{d}, P_{c}\right)$, as seen in Fig. $7(\mathrm{c})$ : since, for both the odd and even SDCS families, $H\left(P_{d}, P_{c}\right)$ is a smooth single-valued surface with no cuspidal edges (Whitney surfaces), neither type of the solitons should change its stability within its existence domain [27, 29].

The enhanced localization of the composite solitons with increasing power $P_{d}$ of the discrete component can be exploited in all-optical beam-steering devices $[4,9,30]$. To demonstrate this possibility, we consider SDCSs with a phase tilt, accounted for by transverse wavenumber $k_{0}$, at the input facet of the waveguide array, $\left.\left\{\phi_{n}, \Psi\right\}\right|_{\zeta=0}=$ $\left\{\bar{\phi}_{n}^{(\mathrm{sol})}, \bar{\Psi}^{(\mathrm{sol})}\right\} e^{i k_{0} \eta}$, and follow their propagation in the waveguide array. Figure 8 presents the results for odd (stable) solitons, in the case of $\lambda_{d}=\lambda_{c}=\lambda$. As might be expected, at low power $P_{d}$ the "kicked" (phase-tilted) SDCS readily moves across the waveguide array. However, as $P_{d}$ increases, the transverse displacements of both the discrete and continuous components decrease, and, for $P_{d}$ exceeding a certain critical value [in Fig. 8, 

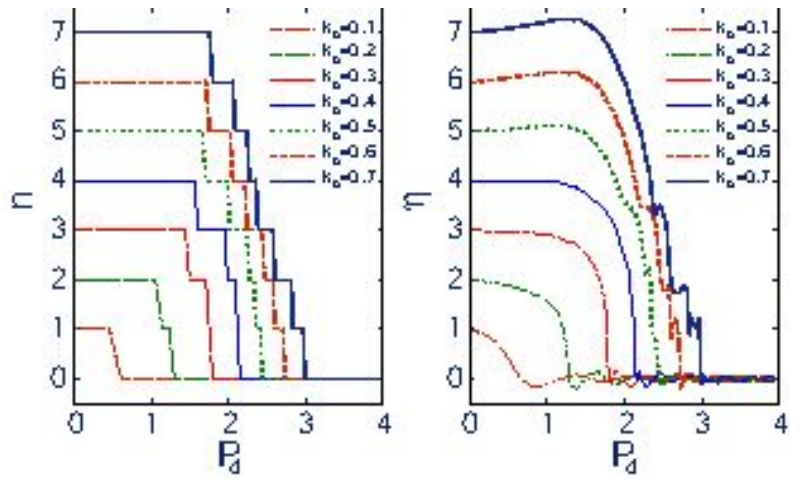

FIG. 9: (Color online) The location of the peak intensity in the discrete and continuous components (left and right panels, respectively) of the odd soliton versus power $P_{d}$ of its discrete component, at different values of phase pitch $k_{0}$ applied at $\zeta=0$. The coupling constant is $\kappa=0.1$.

$\left(P_{d}\right)_{\mathrm{cr}} \approx 2$ and 3, for $k_{0}=0.4$ and 0.7 , respectively], the soliton remains trapped at its initial location. This finding is consistent with the fact that the PNB increases with $P_{d}$, as per Fig. $3(\mathrm{c})$.

Dependencies of the transverse displacement of the odd SDCS on phase pitch $k_{0}$, which causes its displacement, and power $P_{d}$ of its discrete component, are displayed in Fig. 9. Naturally, the transverse displacement increases with $k_{0}$, as well as critical power $\left(P_{d}\right)_{\mathrm{cr}}$ required to pin the soliton. It is worthy to note that the discrete and continuous components remain mutually trapped even close to the linear limit (at small $P_{d}$ ), which is not obvious in Fig. 8.

To summarize, we have demonstrated that the struc- ture built as a single-mode slab waveguide coupled to a discrete waveguide array, with both parts made of a Kerr-nonlinear material, supports semi-discrete composite solitons of odd (onsite-centered), even (intersitecentered), and twisted types. One- and two-parameter families of the odd solitons (with one or two independent wavenumbers, respectively) are stable, while the other types are not. Note that in the case of optical media with quadratic nonlinearity both even and twisted SDCSs may be stable. This should not be a surprise as quadratically and cubically nonlinear media represent essentially different optical system and therefore one expects that they support nonlinear modes with different physical properties. In addition, in the case of quadratically nonlinear media, SDCSs have a more complex structure, as the discrete and the continuous components can be excited at either the fundamental frequency or at the second harmonic.

The potential application of the stable solitons, kicked in the transverse direction, to all-optical beam steering was also demonstrated. The analysis presented here for the one-dimensional setting can be extended to twodimensional lattices and photonic-crystal fibers. For example, nonlinear modes supported by $2 \mathrm{D}$ nonlinear optical media invariant to discrete symmetry transformations have already been investigated [31, 32, 33, 34]. It may also be interesting to analyze semi-discrete solitons in media with the self-defocusing cubic nonlinearity, where the discrete component would be of the staggered type.

This work was supported by NSF under Grant No. ECS-0523386, and by DARPA/AFOSR under Grant No. FA9550-05-1-0428.
[1] D. N. Christodoulides, F. Lederer, and Y. Silberberg, Nature (London) 424, 817 (2003).

[2] D. N. Christodoulides and R. I. Joseph, Opt. Lett. 13, 794 (1988).

[3] Y. S. Kivshar, Opt. Lett. 18, 1147 (1993).

[4] A. B. Aceves, C. De Angelis, T. Peschel, R. Muschall, F. Lederer, S. Trillo, and S. Wabnitz, Phys. Rev. E 53, 1172 (1996).

[5] B. A. Malomed and P. G. Kevrekidis, Phys. Rev. E 64, 026601 (2001).

[6] K. G. Makris, S. Suntsov, D. N. Christodoulides, G. I. Stegeman, and A. Hache, Opt. Lett. 30, 2466 (2005).

[7] D. Mihalache, D. Mazilu, F. Lederer, and Y. S. Kivshar, Opt. Lett. 32, 2091 (2007).

[8] K. Levy and B. A. Malomed, J. Opt. Soc. Am. B 25, 302 (2008).

[9] T. Peschel, U. Peschel, and F. Lederer, Phys. Rev. E 57, 1127 (1998).

[10] J. W. Fleischer, T. Carmon, M. Segev, N. K. Efremidis, and D. N. Christodoulides, Phys. Rev. Lett. 90, 023902 (2003).

[11] H. S. Eisenberg, Y. Silberberg, R. Morandotti, A. R. Boyd, and J. S. Aitchison, Phys. Rev. Lett. 81, 3383
(1998).

[12] H. Zeng, J. Wu, H. Xu, and K. Wu, Phys. Rev. Lett. 96, 083902 (2006).

[13] N. C. Panoiu, R. M. Osgood, and B. A. Malomed, Opt. Lett. 31, 1097 (2006).

[14] V. R. Almeida, R. R. Panepucci, and M. Lipson, Opt. Lett. 28, 1302 (2003).

[15] S. Imamura, R. Yoshimura, and T. Izawa, Electron. Lett. 27, 1342 (1991).

[16] W. T. Tsang and R. A. Logan, IEEE J. Quantum Electron. 15, 451 (1979).

[17] E. Dulkeith, F. Xia, L. Schares, W. M. J. Green, and Y. A. Vlasov, Opt. Express 14, 3853 (2006).

[18] K. P. Panajotov and A. T. Andreev, J. Opt. Soc. Am. B 11, 826 (1994).

[19] S. M. Jensen, IEEE J. Quantum Electron. 18, 1580 (1982); G. I. Stegeman, IEEE J. Quantum Electron. 18, 1610 (1982).

[20] A. M. Maier, Kvantovaya Elektron. (Moscow) 9, 2296 (1982); [Sov. J. Quant. Electr. 12, 1490 (1982)].

[21] D. J. Kaup, B. A. Malomed, and R. S. Tasgal, Phys. Rev. E 48, 3049 (1993).

[22] M. Haelterman, A. P. Sheppard, and A. W. Snyder, Opt. 
Lett. 18, 1406 (1993).

[23] J. Hudock, P. G. Kevrekidis, B. A. Malomed, and D. N. Christodoulides, Phys. Rev. E 67, 056618 (2003).

[24] Y. A. Kivshar and D. K. Campbell, Phys. Rev. E 48, 3077 (1993).

[25] M. G. Vakhitov and A. A. Kolokolov, Radiophys. Quant. Electr. 16, 783 (1973).

[26] A. V. Buryak, Y. S. Kivshar, and S. Trillo, Phys. Rev. Lett. 77, 5210 (1996).

[27] A. V. Buryak and Y. S. Kivshar, Phys. Rev. Lett. 78, 3286 (1997).

[28] N. C. Panoiu, D. Mihalache, H. Rao, and R. M. Osgood, Phys. Rev. E 68, 065603(R) (2003).
[29] F. V. Kusmartsev, Phys. Rep. 183, 1 (1989).

[30] N. C. Panoiu, M. Bahl, and R. M. Osgood, Opt. Lett. 28, 2503 (2003); ibid., J. Opt. Soc. Am. B 21, 1500 (2004).

[31] S. F. Mingaleev, Y. S. Kivshar, and R. A. Sammut, Phys. Rev. E 62, 5777 (2000).

[32] N. C. Panoiu, M. Bahl, and R. M. Osgood, Opt. Express 12, 1605 (2004).

[33] G. Van der Sande, B. Maes, P. Bienstman, J. Danckaert, R. Baets, and I. Veretennicoff, Opt. Express 13, 1544 (2005).

[34] J. R. Salgueiro, Y. S. Kivshar, D. E. Pelinovsky, V. Simon, and H. Michinel, St. Appl. Math. 115, 157 (2005). 